\title{
Development of a RILIS ionisation scheme for gold at ISOLDE, CERN
}

\author{
B. A. Marsh • V. N. Fedosseev • P. Kosuri • ISOLDE \\ Collaboration
}

Published online: 15 February 2007

(C) Springer Science + Business Media B.V. 2007

\begin{abstract}
At the ISOLDE on-line isotope separation facility, the resonance ionisation laser ion source (RILIS) can be used to ionise reaction products as they effuse from the target. The RILIS process of laser step-wise resonance ionisation of atoms in a hot metal cavity provides a highly element selective stage in the preparation of the radioactive ion beam. As a result, the ISOLDE mass separators can provide beams of a chosen isotope with greatly reduced isobaric contamination. With the addition of a new three-step ionisation scheme for gold, the RILIS is now capable of ionising 26 of the elements. The optimal scheme was determined during an extensive study of the atomic energy levels and auto-ionising states of gold, carried out by means of in-source resonance ionisation spectroscopy. Details of the ionisation scheme and a summary of the spectroscopy study are presented.
\end{abstract}

Key words radioactive ion beams • resonance ionisation laser ion source $\cdot \mathrm{Au}$

\section{Introduction}

ISOLDE is an isotope separator on-line (ISOL) type radioactive ion beam facility. Radionuclide production occurs when a pulsed proton beam of 1 or $1.4 \mathrm{GeV}$ is incident upon a thick target. A multitude of reaction channels (e.g. spallation, fragmentation and fission) contribute to the production of a wide spectrum of isotopes.

B. A. Marsh (凶) · V. N. Fedosseev

CERN, CH-1211, Geneva-23, Switzerland

e-mail: bruce.marsh@cern.ch

P. Kosuri

KTH, Stockholm SE-100 44, Sweden

B. A. Marsh

The University of Manchester, Manchester M13 9PL, United Kingdom 
Recoiling reaction products are stopped within a short distance inside the heated target material and so their transit to the ion source is by means of diffusion and effusion processes. Unambiguous isotope separation relies on the availability of the reaction products in a single charge state and with a low velocity spread. At ISOLDE, $1^{+}$ions are most commonly produced and are subject to a $60 \mathrm{keV}$ accelerating voltage. There are two target stations, each connected to its own magnetic dipole mass separator: the General Purpose Separator (GPS) or the High Resolution Separator (HRS). The respective resolving powers of $M / \Delta M \approx 2,500$ and $M / \Delta M \approx$ 10,000 are sufficient for the selection of a single atomic mass for transmission. For the chosen atomic mass, often many isobars are produced and isotope selection is not possible by mass separation alone. The somewhat conventional techniques of temperature optimization and beam gating are at hand to give preferential release of the desired element however, such measures are not universally applicable and are often limited in success. The Resonance Ionisation Laser Ion Source (RILIS) combines an unsurpassed level of $Z$-selectivity with a rapid and efficient ionisation process $[1,2]$. For RILIS, ionisation is achieved through a step-wise resonance photon absorption process, providing a high degree of selectivity by exploiting the unique electronic structure of different atomic species. In principle, the RILIS can be used for the ionisation of almost all metallic elements that are released from the ISOLDE target. An important aspect of ongoing development is the extension of its range with the study of resonance ionisation schemes. Recently a request for the development of gold ion beams was addressed to the ISOLDE and Neutron Time of Flight Experiments Committee (INTC) by the letter of intent 'Study of the $\pi h_{11 / 2}^{-1}$ isomeric states in ${ }^{201,203,205} \mathrm{Au}$. Following an endorsement of this intent by the committee, the task of finding an efficient RILIS ionisation scheme for gold was put forward.

Resonance ionisation spectroscopy has been applied for gold by several groups. The data obtained as well as the ionisation schemes used are compiled by Saloman [3]. In particular, an efficient ionisation scheme with a three-colour, three-step excitation to an autoionising state has been used for on-line laser spectroscopy of laser-desorbed gold isotopes [4, 5]. Auto-ionising (AIS) states of Au were also studied by resonance ionisation spectroscopy in $[6,7]$. Since the RILIS laser system and the conditions in the ionisation region are different from those used in $[4,6,7]$, investigating the performance of these known schemes at the RILIS setup was necessary. In addition, the search for transitions to the auto-ionising states was carried out in these works for only a few excited states in a limited spectral range, which is not readily available at RILIS. Therefore, a new, more extensive study of gold resonance ionisation at the RILIS setup was required for defining the optimal ionisation scheme. In this article we present results of the resonance ionisation spectroscopy study of gold atomic transitions performed at the RILIS setup. Based on the experimental results, the optimal ionisation scheme is defined. A summary is presented along with details of this new ionisation scheme.

\section{The resonance ionisation laser ion source}

References [2, 8, 9] give a thorough description of the ISOLDE RILIS. A master oscillator power amplifier system of copper vapor lasers (CVL) operating at the 
pulse repetition rate of $11 \mathrm{kHz}$ provides two output laser beams, each with an average power of typically $30-40 \mathrm{~W}$. The RILIS set-up includes three dye lasers and therefore ionisation schemes employing up to three resonant transitions can be used. The wavelength range of the dye lasers is 530-850 $\mathrm{nm}$. Frequency doubling and tripling of dye laser beams are carried out using non-linear BBO (beta-barium borate) crystals to generate 2 nd or 3 rd harmonics of the fundamental beam, extending the wavelength range to include $214-415 \mathrm{~nm}$. This enables high lying first excited states to be attained and is essential for elements with a high ionisation potential. With this current work included, the RILIS has been used for resonance ionisation of 26 of the elements. Schemes using one, two or three resonant transitions have been used. Most commonly, the ionisation step is a transition to the continuum using an available CVL beam. Alternatively, the final step can be a resonant transition to an auto-ionising state. A transition to an auto-ionising state can have a high cross section, which is favorable for improving the ionisation efficiency. Ionisation takes place in a hot cavity connected to the target. Reaction products enter this cavity as an atomic vapor at a temperature of around $2,300 \mathrm{~K}$. The role of the cavity is to contain the atoms for a certain time within a volume where they can be irradiated by the laser light and to confine the ions during their drift towards the extraction region. The ionisation cavities are refractory metal ( $\mathrm{W}$ or $\mathrm{Nb}$ ) tubes with an inner diameter of $3 \mathrm{~mm}$ and a length of typically $30 \mathrm{~mm}$. They are resistively heated to a temperature of about 2,300 K with a DC current of 200-350 A. After leaving the source, ions are accelerated to $60 \mathrm{kV}$, separated in a magnetic field and guided by electrostatic ion-optical elements to the experimental setup.

For this work, a standard ISOLDE target was used with a tungsten surface ioniser cavity. The target/ion source unit was equipped with two ovens. A large gold sample was placed in one of these, for use during the initial spectroscopy study and ionisation scheme search. The second oven was loaded with a precise 3,000 nAh gold sample, for use during the final efficiency measurement. The mass separator was tuned to the mass of ${ }^{197} \mathrm{Au}$ and the transmitted ion current was monitored on a Faraday cup detector.

\section{Resonance ionisation spectroscopy of gold}

Data on atomic lines and energy levels for gold are taken from the Kurucz atomic line database (http://cfa-www.harvard.edu/amdata/ampdata/kurucz23/sekur.html). The ionisation potential for gold $(9.23 \mathrm{eV})$ is high with respect to the photon energyrange attainable with RILIS $(\sim 1.7-5 \mathrm{eV})$. As a result, ionisation schemes using three excitation steps were investigated. For the first step, the transitions from the ground state $6 s^{2} \mathrm{~S}_{1 / 2}$ level to the $6 p^{2} \mathrm{P}_{1 / 2}$ and $6 p^{2} \mathrm{P}_{3 / 2}$ levels at $37,358.991 \mathrm{~cm}^{-1}$ and $41,174.613 \mathrm{~cm}^{-1}$ respectively are the only known transitions that can be reached with the RILIS laser system. Both transitions are strong and of similar strength $(A=1.65$. $10^{8} \mathrm{~s}^{-1}$ and $\left.1.96 \cdot 10^{8} \mathrm{~s}^{-1}\right)$. At $242.9 \mathrm{~nm}$, the wavelength required to populate the $41,174.61 \mathrm{~cm}^{-1}$ level is at the short wavelength end of the RILIS tuning range and can be generated only by tripling the fundamental frequency of the dye laser. The efficiency of 3rd harmonic generation is low ( $~ 5 \%$ for a $5 \mathrm{~W}$ fundamental beam) and so, if this transition is used, a large proportion of the CVL pump beam power must be used to obtain the dye laser power required at the fundamental frequency. 

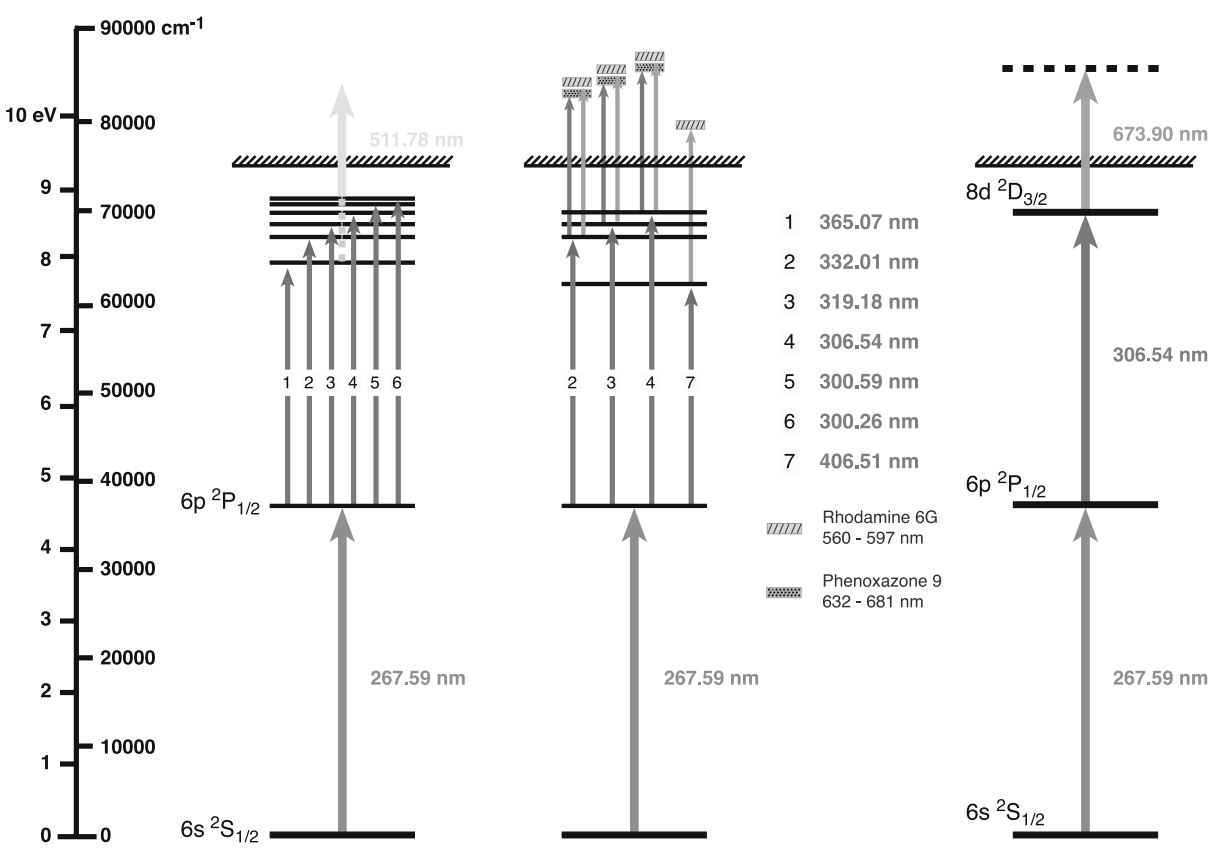

Fig. 1 The scope of the Au RIS study and the optimal ionisation scheme (RHS)

The transition to the lower lying, ${ }^{2} \mathrm{P}_{1 / 2}$ level is preferable since the transition energy, $37,358.991 \mathrm{~cm}^{-1}$, corresponds to that of a $267.673 \mathrm{~nm}$ photon, accessible by $2 \mathrm{nd}$ harmonic generation of light at $535.198 \mathrm{~nm}$. This falls within the emission range of the Pyrromethene 546 dye, which is pumped with the green component of the CVL beam. Measurements were carried out using only this transition for the first step in the excitation scheme.

From this first excited state, 11 potential second excited states from $54,485 \mathrm{~cm}^{-1}$ to $72,164 \mathrm{~cm}^{-1}$ are documented (http://cfa-www.harvard.edu/amdata/ampdata/ kurucz23/sekur.html). All but the lowest lying of these excited states exist at a energy of less than $19,581 \mathrm{~cm}^{-1}$ below the continuum, meaning that ionisation from these levels is possible via non-resonant absorption of a $511 \mathrm{~nm}$ photon, provided by the green CVL beam. Accessing any of these 10 transitions requires 2 nd harmonic generation of the fundamental dye laser beam, and hence, a significant proportion of CVL pump power. It is preferable to use all of the CVL pump power at $511 \mathrm{~nm}$ for the generation of the frequency for the 1st transition and for the final ionisation stage, leaving only the yellow $(578 \mathrm{~nm})$ component for the second step. Therefore, we have limited our study to the group of six second step transitions (Fig. 1), which are accessible with a frequency doubled dye laser pumped with light at $578 \mathrm{~nm}$. The pumping requirements of the dye lasers and amplifiers for the first and second step transitions were met using the total output of the higher power of the two CVL amplifiers after separation of the yellow and green components of the beam. Typical values of the average power for this CVL beam were $22 \mathrm{~W}$ and $20 \mathrm{~W}$ at $511 \mathrm{~nm}$ and $578 \mathrm{~nm}$, respectively. The second CVL beam with a total power (in both components) of $27 \mathrm{~W}$ was available for the last excitation step. 
Table 1 Gold 3 step ionisation schemes

\begin{tabular}{|c|c|c|c|c|c|c|c|}
\hline \multirow{2}{*}{$\begin{array}{l}\lambda_{2} \text { (air) } \\
\mathrm{nm}\end{array}$} & \multirow{2}{*}{$\begin{array}{l}E_{2} \\
\mathrm{~cm}^{-1}\end{array}$} & \multirow[t]{2}{*}{ State II } & \multirow{2}{*}{$\begin{array}{l}\lambda_{3}(\text { air }) \\
\mathrm{nm}\end{array}$} & \multirow{2}{*}{$\begin{array}{l}E_{3} \\
\mathrm{~cm}^{-1}\end{array}$} & \multicolumn{2}{|c|}{ Laser Power, $\mathrm{mW}$} & \multirow[t]{2}{*}{$R$} \\
\hline & & & & & 2 & 3 & \\
\hline 365.07 & $64,742.9$ & $8 s^{2} S_{1 / 2}$ & 511,578 & Continuum & 75 & 10,000 & 1 \\
\hline 332.01 & $67,469.68$ & $7 d^{2} \mathrm{D}_{3 / 2}$ & 511,578 & Continuum & 75 & 10,000 & 9.6 \\
\hline 319.18 & $68,680.63$ & $9 s^{2} \mathrm{~S}_{1 / 2}$ & 511,578 & Continuum & 60 & 10,000 & 2.1 \\
\hline 306.54 & $69,971.42$ & $8 d^{2} \mathrm{D}_{3 / 2}$ & 511,578 & Continuum & 75 & 11,000 & 7.7 \\
\hline 300.59 & $70,617.73$ & $10 s^{2} \mathrm{~S}_{1 / 2}$ & 511,578 & Continuum & 65 & 11,000 & 1.5 \\
\hline 300.26 & $70,653.25$ & $J=3 / 2$ & 511,578 & Continuum & 65 & 11,000 & 1.2 \\
\hline 406.51 & $61,951.89$ & $6 d^{2} D_{3 / 2}$ & 591.90 & $78,842.0$ & 75 & 625 & 55 \\
\hline 319.18 & $68,680.63$ & $9 s^{2} S_{1 / 2}$ & 668.65 & $83,631.9$ & 90 & 1130 & 4.4 \\
\hline 319.18 & $68,680.63$ & $9 s^{2} \mathrm{~S}_{1 / 2}$ & 644.93 & $84,181.9$ & 90 & 1130 & 3.1 \\
\hline 332.01 & $67,469.68$ & $7 d^{2} \mathrm{D}_{3 / 2}$ & 592.85 & $84,332.8$ & 90 & 500 & 10 \\
\hline 332.01 & $67,469.68$ & $7 d^{2} \mathrm{D}_{3 / 2}$ & 584.84 & $84,563.8$ & 90 & 750 & 6.4 \\
\hline 332.01 & $67,469.68$ & $7 d^{2} \mathrm{D}_{3 / 2}$ & 578.38 & $84,754.5$ & 90 & 550 & 73 \\
\hline 306.54 & $69,971.42$ & $8 d^{2} \mathrm{D}_{3 / 2}$ & 676.27 & $84,754.3$ & 80 & 750 & 150 \\
\hline 332.01 & $67,469.68$ & $7 d^{2} \mathrm{D}_{3 / 2}$ & 576.64 & $84,806.6$ & 90 & 950 & 120 \\
\hline 306.54 & 69,971.42 & $\mathbf{8} \boldsymbol{d}^{2} \mathrm{D}_{3 / 2}$ & 673.9 & $84,806.3$ & 80 & 750 & 220 \\
\hline 332.01 & $67,469.68$ & $7 d^{2} \mathrm{D}_{3 / 2}$ & 574.20 & $84,880.5$ & 90 & 750 & 34 \\
\hline 306.54 & $69,971.42$ & $8 d^{2} \mathrm{D}_{3 / 2}$ & 670.55 & $84,880.4$ & 80 & 1000 & 180 \\
\hline 306.54 & $69,971.42$ & $8 d^{2} \mathrm{D}_{3 / 2}$ & 666.24 & $84,976.9$ & 80 & 1130 & 13 \\
\hline 332.01 & $67,469.68$ & $7 d^{2} \mathrm{D}_{3 / 2}$ & 570.77 & $84,985.0$ & 90 & 750 & 2.6 \\
\hline 306.54 & $69,971.42$ & $8 d^{2} \mathrm{D}_{3 / 2}$ & 665.81 & $84,986.6$ & 80 & 1130 & 38 \\
\hline 332.01 & $67,469.68$ & $7 d^{2} \mathrm{D}_{3 / 2}$ & 569.80 & $85,014.7$ & 90 & 975 & 3.5 \\
\hline 332.01 & $67,469.68$ & $7 d^{2} \mathrm{D}_{3 / 2}$ & 567.24 & $85,094.1$ & 90 & 600 & 4.5 \\
\hline 306.54 & $69,971.42$ & $8 d^{2} \mathrm{D}_{3 / 2}$ & 661.08 & $85,093.9$ & 80 & 1250 & 21 \\
\hline 332.01 & $67,469.68$ & $7 d^{2} \mathrm{D}_{3 / 2}$ & 567.12 & $85,097.8$ & 90 & 600 & 5.4 \\
\hline 306.54 & $69,971.42$ & $8 d^{2} \mathrm{D}_{3 / 2}$ & 660.90 & $85,098.0$ & 80 & 1250 & 20 \\
\hline 332.01 & $67,469.68$ & $7 d^{2} \mathrm{D}_{3 / 2}$ & 564.14 & $85,190.8$ & 90 & 450 & 3.3 \\
\hline 306.54 & $69,971.42$ & $8 d^{2} \mathrm{D}_{3 / 2}$ & 632.80 & $85,769.9$ & 80 & 500 & 4.3 \\
\hline 319.18 & $68,680.63$ & $9 s^{2} S_{1 / 2}$ & 567.39 & $86,300.3$ & 80 & 750 & 4.5 \\
\hline 319.18 & $68,680.63$ & $9 s^{2} S_{1 / 2}$ & 565.61 & $86,355.6$ & 85 & 625 & 3.8 \\
\hline 319.18 & $68,680.63$ & $9 s^{2} \mathrm{~S}_{1 / 2}$ & 565.36 & $86,363.5$ & 85 & 625 & 3.6 \\
\hline 306.54 & $69,971.42$ & $8 d^{2} \mathrm{D}_{3 / 2}$ & 575.60 & $87,339.7$ & 85 & 750 & 76 \\
\hline 306.54 & $69,971.42$ & $8 d^{2} \mathrm{D}_{3 / 2}$ & 572.38 & $87,437.6$ & 85 & 750 & 59 \\
\hline 306.54 & $69,971.42$ & $8 d^{2} \mathrm{D}_{3 / 2}$ & 570.70 & $87,488.4$ & 85 & 750 & 59 \\
\hline 306.54 & $69,971.42$ & $8 d^{2} \mathrm{D}_{3 / 2}$ & 567.98 & $87,572.8$ & 85 & 700 & 44 \\
\hline 306.54 & $69,971.42$ & $8 d^{2} \mathrm{D}_{3 / 2}$ & 567.14 & $87,598.7$ & 85 & 550 & 7.4 \\
\hline 306.54 & $69,971.42$ & $8 d^{2} \mathrm{D}_{3 / 2}$ & 564.07 & $87,694.9$ & 85 & 550 & 15 \\
\hline 306.54 & $69,971.42$ & $8 d^{2} \mathrm{D}_{3 / 2}$ & 561.71 & $87,769.1$ & 85 & 375 & 4.3 \\
\hline
\end{tabular}

$E_{0}=6 s^{2} \mathrm{~S}_{1 / 2}, 0 \mathrm{~cm}^{-1} ; E_{1}=6 p^{2} \mathrm{P}_{1 / 2}, 37,358.99 \mathrm{~cm}^{-1}$

The laser power was measured on the laser table, transmission to the ion source is approximately $20 \%$ for the first step, $50 \%$ for the second step and $50 \%$ for the CVL beam (3rd step)

As the first stage of this study, schemes using 3-step ionisation with a non-resonant transition to the continuum (induced by both the green and the yellow components of the CVL beam) were studied. During this study, a ion current of gold was observed in the presence of only the beam for the first step in the excitation scheme. Since the ionisation threshold of gold $\left(74,409.0(2) \mathrm{cm}^{-1}[10]\right)$ is slightly less than the sum 

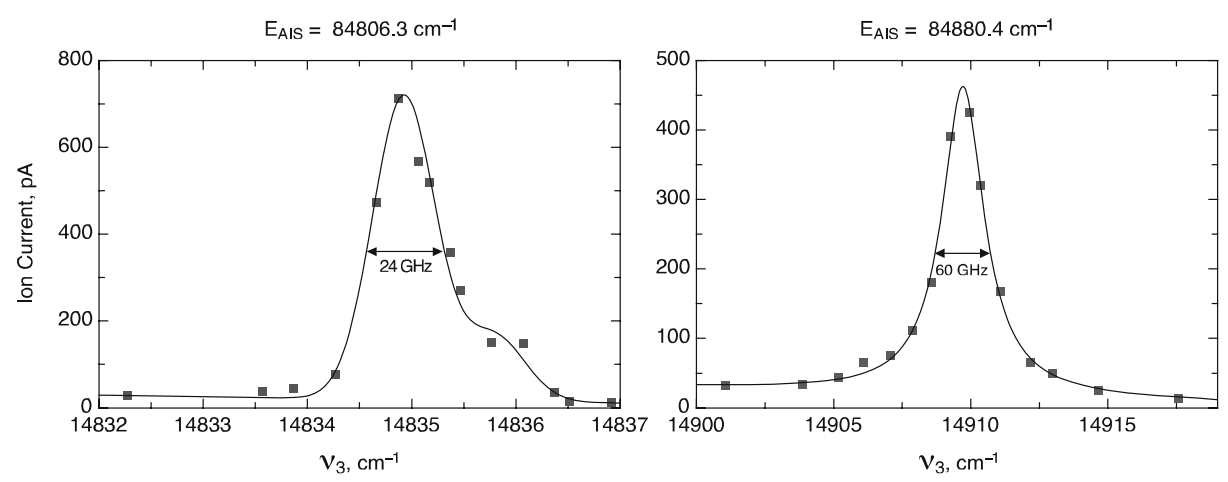

Fig. 2 The two strongest AIS resonances observed

of the energies of two photons at $267.673 \mathrm{~nm}$, this ion current is attributed to the occurrence of two-step ionisation via a transition to the continuum from the first excited state by non-resonant absorption of second photon from the first step beam. The presence of this laser-ionised ion signal facilitated a reliable optimization of the beam focusing and positioning. In addition, the measurements of the ion current produced by the first step transition beam provided a useful reference point for use in the evaluation of the relative efficiencies of ionisation schemes. The ratio of ion current produced with three laser beams versus ion current produced by the first step beam $R=I_{1+2+3} / I_{1+3}$ is taken as an efficiency parameter of the ionisation scheme. In order to keep thermal conditions of laser beam transport optics constant these measurements were carried out whilst blocking only the beam of second step laser, leaving the most powerful laser beam propagating toward the ion source. This beam did not participate in the ionisation process because its photon energy was less than required for transition from the first excited state to the ionisation continuum.

For the second phase of the study a search for auto-ionising states was conducted. The CVL power used for the ionisation stage was diverted to pump a 3rd dye laser and dye amplifier. Ethanol solutions of the laser dyes Rhodamine 6G and Phenoxazone 9 were used in this laser, respectively the wavelength was scanned in the ranges of 560-597 $\mathrm{nm}$ and $632-681 \mathrm{~nm}$. When an observable increase of the ion current was detected, scanning was stopped and the wavelength was optimized to the maximal value of the ion current. Its peak value, the corresponding transition frequency, and the values of laser power for each of the three beams were recorded. Measurements of the laser frequency were made with a HighFinesse / Angstrom WS/7 wavemeter. The accuracy of the transition frequency measurements was defined by the width of resonance and laser line width $\left(\sim 0.6 \mathrm{~cm}^{-1}\right)$. For narrow AIS it was possible to define the peak position with the accuracy of about $0.1 \mathrm{~cm}^{-1}$. Energy regions above the ionisation threshold for the transitions from the second excited states corresponding to the four strongest second step transitions were studied.

A summary of these measurements is given in Table 1. Within the energy intervals that were studied, five auto-ionising states had been previously observed [4, 7], but not as resonant transitions from the excited states measured during this study. Three of the known AIS were confirmed and 27 new AIS were observed. Figures $2 \mathrm{a}$ and $2 \mathrm{~b}$ show the profiles of the resonances for the two strongest AIS, at $84,806.3 \mathrm{~cm}^{-1}$ 

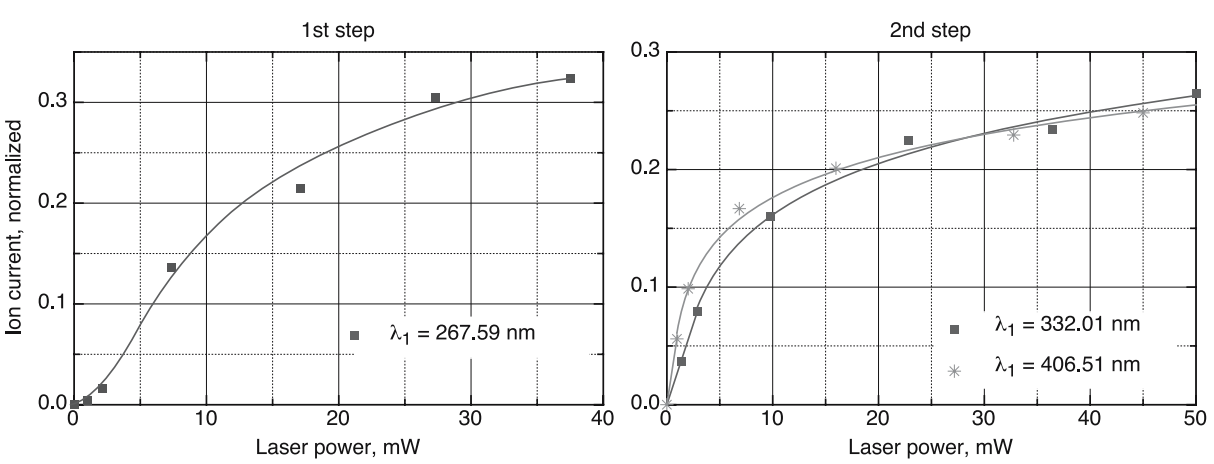

Fig. 3 Saturation curves for the resonant transitions of the most efficient Au schemes

and $84,880.4 \mathrm{~cm}^{-1}$, respectively. Of particular interest was the measurement of a previously applied ionisation scheme for gold [4]. This scheme, shown in italics in Table 1, was one of the more efficient schemes previously measured however the optimum scheme, shown on the right hand side of Fig. 1, gives a factor of 4-5 increase in the efficiency parameter $R$. The saturation measurements for the 1 st and 2 nd step in this scheme are presented in Fig. 3, they confirm that the saturation points for the transitions are exceeded with the laser power available.

For the measurement of the absolute value of ionisation efficiency, some experimental difficulties were encountered, limiting the reliability of the assessment of the mass marker evaporation. During the initial search for a resonance ionisation signal, a large quantity of the gold sample was evaporated from the first oven. After detection of the ion signal, the subsequent tests were carried out without the need to heat the mass marker itself, evaporation of the gold that had condensed in the target container provided a sufficient and steady supply of gold vapor for the duration of the spectroscopic work. An attempt was made to extinguish this supply by gradually increasing the target heating from 570-700 A. A small decrease in the ion current was observed after some hours. The evaporation of the 3,000 nAh mass marker was then carried out with the target heating kept at 700 A. For improved laser ionisation stability and experimental ease, only the first step laser was used for ionisation during the efficiency measurement. The ionisation efficiency of the scheme presented in Fig. 1 was estimated to be over $3 \%$ by comparing the product of the ion current integration and the parameter $R$ of the most efficient ionisation scheme with the original sample size.

\section{Conclusion}

With the development of this new ionisation scheme for gold, the RILIS can now provide a means of efficient and selective ionisation of 26 of the elements. In accordance with the requirements of the ISOLDE experimental program, new schemes will continue to be developed for RILIS using procedures similar to those described in this work. Currently, such studies have to fit into the framework of general ISOLDE off-line development and thus, the dedicated mass separator use required is limited to just a few weeks per year. A recent letter of intent addressed to 
the INTC, 'Development of the RILIS research laboratory at ISOLDE' gives details of a new and dedicated RILIS spectroscopy facility. Following its completion, the laboratory will enable the investigation of new ionisation schemes as a task that is independent of ISOLDE scheduling. Many of the RILIS ionisation schemes rely on an inefficient final step of non-resonant ionisation using a large proportion of the total CVL power. It is hoped that the time consuming search for auto-ionising states for many of these elements could be carried out in this laboratory and significant improvements in RILIS efficiency could be made.

Acknowledgements The target preparation (assembly, mass marker preparation and initial testing) was carried out by R. Catherall, D. Carminati, B. Crepieux and T. Stora (CERN-AB). This work was supported by the EU 6th programme Integrating Infrastructure Initiative Transnational Access, Contract number: RII3-CT-2004-506065.

\section{References}

1. Mishin, V.I., Fedoseyev, V.N., Kluge, H.-J., Letokhov, V.S., Ravn, H.L., Scheerer, F., Shirakabe, Y., Sundell, S., Tengblad, O., ISOLDE Collaboration: Nucl. Instrum. Methods Phys. Res., B 73, 550 (1993)

2. Fedoseyev, V.N., Huber, G., Köster, U., Lettry, J., Mishin, V.I., Ravn, H.L., Sebastian, V., ISOLDE Collaboration: Hyperfine Interact. 127, 409 (2000)

3. Saloman, E.B.: Spectrochim. Acta, Part B 45, 37 (1990)

4. Krönert, U., Beckert, St., Hilberath, Th., Kluge, H.-J., Schulz, C.: Appl. Phys., A 44, 339 (1987)

5. Krönert, U., Beckert, St., Bollen, G., Gerber, M., Hilberath, Th., Kluge, H.-J., Passler, G., ISOLDE Collaboration: Nucl. Instrum. Methods Phys. Res., A 300, 522 (1991)

6. Bekov, G.I., Tursunov, A.T., Khasanov, G., Eshkobilov, N.B.: Opt. Spectrosc. (USSR) 62, 163 (1987)

7. Zhao, W.Z., Xu, X.Y., Ma, W.Y., Cheng, Y., Hui, Q., Wen, K.L., Chen, D.Y.: Appl. Phys., B 52, 299 (1991)

8. Fedosseev, V.N., Fedorov, D.V., Horn, R., Huber, G., Köster, U., Lassen, J., Mishin, V.I., Seliverstov, M.D., Weissman, L., Wendt, K., ISOLDE Collaboration: Nucl. Instrum. Methods Phys. Res., B 204, 353 (2003)

9. Catherall, R., Fedosseev, V.N., Köster, U., Lettry, J., Suberlucq, G., Marsh, B.A., Tengborn, E., ISOLDE Collaboration: Rev. Sci. Instrum. 75, 1614 (2004)

10. Loock, H.-P., Beaty, L.M., Simard, B.: Phys. Rev., A 59, 873 (1999) 